\title{
Meshless implementations of local integral equations
}

\author{
V. Sladek ${ }^{1}$, J. Sladek ${ }^{1}$ \& Ch. Zhang $^{2}$ \\ ${ }^{1}$ Institute of Construction and Architecture, \\ Slovak Academy of Sciences, Slovakia \\ ${ }^{2}$ Department of Civil Engineering, University of Siegen, Germany
}

\begin{abstract}
The paper deals with the development of the meshless computational techniques based on the Local Integral Equations and analytical integrations. The development is illustrated on 2-d potential problems in functionally graded media. The MLS-approximation is used for simulation of spatial variations of the potential field. Efficient differentiation is proposed for calculation of derivatives of shape functions. The accuracy and efficiency are studied on simple example providing the exact solution for the benchmark solution.
\end{abstract}

Keywords: MLS-approximation, local integral equations, differentiation of shape functions, accuracy, efficiency.

\section{Introduction}

The advantages of mesh-free formulations in comparison with the mesh-based ones have been appreciated extensively in solution of problems for separable media. In two last decades, many mesh-free formulations are becoming popular also in numerical analyses for solids because of their high adaptivity and a low cost preparation of input data. A variety of meshless approximations have been implemented in discretizations of various formulations for numerical solution of boundary value problems. The application of the weak formulation on local subdomains enables development of truly mesh-free formulations in contrast to the weak formulations considered in the global sense, where the background mesh is still required. Nevertheless, there is a criticism as regards the time requirements for evaluation of the shape functions in various meshless approximations. This is so owing to rather complicated form of the shape functions, hence certain algorithm is to be repeated for evaluation of them at each integration point. 
In this paper, we shall consider Moving Least Squares (MLS) approximations [1] in potential problems. Then, the physical field values are expressed in terms of certain nodal unknowns which are different from the nodal values of the approximated field, in general. The nodes playing the role in approximation of the potential field at certain point $\mathbf{x}$ are selected by weight functions associated to nodes. For each integration point $\mathbf{x}$, it is necessary to find if the weight function is different from zero at $\mathbf{x}$. Certain time savings can be achieved by using MLS-CAN formulation [2], where the Central Approximation Node is taken as the nearest node to the point $\mathbf{x}$ and the nodes influencing the approximation at the CAN are employed as the nodes contributing to the approximation at the point $\mathbf{x}$ too. Recall that the nodes associated with each CAN are specified in advance and saved in memory.

In this paper, we develop a weak formulation for solution of potential problems based on the local integral equations and several kinds of approximations for derivatives of the potential field represented by the MLSapproximation. Performing the integrations analytically, the amount of evaluations of the shape functions is drastically reduced, since these are constrained to nodal points alone. Thus, the computational effort resembles that in the finite difference method. The relationship with the strong formulation based on the collocation of the partial differential equation at nodal points is discussed too. Consideration of the material non-homogeneity does not give rice to any complication as compared with the homogeneous case. The accuracy and computational efficiency is studied in numerical experiments.

\section{Local integral equation formulation for solution of b.v.p.}

The potential problem (e.g. stationary heat conduction) in anisotropic and continuously non-homogeneous media is governed by the following partial differential equation with variable coefficients [3]

$$
\left(\lambda_{i k}(\mathbf{x}) u_{, k}(\mathbf{x})\right)_{, i}=-Q(\mathbf{x}), \text { in } \Omega
$$

where $u(\mathbf{x})$ is the unknown potential field, $Q(\mathbf{x})$ is the known body source density, and $\lambda_{i k}(\mathbf{x})$ describe the spatial variation of the material coefficients related to the flux vector $q_{i}(\mathbf{x})$ as

$$
q_{i}(\mathbf{x})=-\lambda_{i k}(\mathbf{x}) u_{, k}(\mathbf{x})
$$

Physically, Eq. (2) is known as the Fourier law for heat conduction or also as the first Fick's law in diffusion problems.

The prescribed boundary conditions (b.c.) can be classified as

(i) Dirichlet b.c.: $u(\mathbf{x})=\bar{u}(\mathbf{x})$ at $\mathbf{x} \in \partial \Omega_{D}$

(ii) Neumann b.c.: $n_{i}(\mathbf{x}) q_{i}(\mathbf{x})=\bar{q}(\mathbf{x})$ at $\mathbf{x} \in \partial \Omega_{N}$ 
(iii) Robin b.c.: $\alpha u(\mathbf{x})+\beta n_{i}(\mathbf{x}) q_{i}(\mathbf{x})=0$ at $\mathbf{x} \in \partial \Omega_{R},(\alpha, \beta \in R)$

where $\partial \Omega=\partial \Omega_{D} \cup \partial \Omega_{N} \cup \partial \Omega_{R}, n_{i}(\mathbf{x})$ is the unit outward normal vector to the boundary, and an over bar denotes the prescribed quantities.

Recall that Eq. (1) is the differential form (strong form) of the of the energy balance and can be deduced from its integral form

$$
\int_{\partial \Omega^{c}} n_{i}(\mathbf{x}) q_{i}(\mathbf{x}) d \Gamma(\mathbf{x})=\int_{\Omega^{c}} Q(\mathbf{x}) d \Omega(\mathbf{x}),
$$

holding for all arbitrary but small material domains $\Omega^{c} \subset \Omega$. In view of Eq. (2), we can rewrite Eq. (3) as

$$
\int_{\partial \Omega^{c}} n_{i}(\mathbf{x}) \lambda_{i k}(\mathbf{x}) u_{, k}(\mathbf{x}) d \Gamma(\mathbf{x})=-\int_{\Omega^{c}} Q(\mathbf{x}) d \Omega(\mathbf{x}),
$$

which is the local weak form of the governing equations. Note that Eq. (4) is a physically admissible constraint that can be used as a coupling equation in the computation of unknown degrees of freedom of the discretized problem. Recall that the local integral equations (4) are non-singular, since there are no singular fundamental solutions involved in contrast to the singular integral equations employed in the boundary integral equation method. Moreover, the integration of unknown (approximated) field variables is constraint to the boundary of local sub-domains even in the case of non-homogeneous media. This can be effectively utilized by decreasing the amount of integration points as compared with the formulations involving domain integrals. As regards the computational time, it is independent on the fact if the medium is homogeneous or nonhomogeneous.

\section{Moving Least Squares approximation (MLS)}

\subsection{Standard MLS approximation}

The primary field variable (potential field) is assumed to be approximated at a vicinity of the point $\mathbf{x}$ as

$$
u(\mathbf{x}) \approx \sum_{\mu=1}^{m} p_{\mu}(\mathbf{x}) c_{\mu}(\mathbf{x}),
$$

where $\left\{p_{1}(\mathbf{x}), \ldots, p_{m}(\mathbf{x})\right\}$ is a complete monomial basis and $c_{\mu}(\mathbf{x})$ are expansion coefficients which can be obtained by minimizing a weighted functional

$$
J(\mathbf{x})=\sum_{a=1}^{N_{t}} w^{a}(\mathbf{x})\left[p_{\mu}\left(\mathbf{x}^{a}\right) c_{\mu}(\mathbf{x})-\hat{u}^{a}\right]\left[p_{\mu}\left(\mathbf{x}^{a}\right) c_{\mu}(\mathbf{x})-\hat{u}^{a}\right] .
$$


Hence, one can get the expansion coefficients and the approximation (5) becomes

$$
u(\mathbf{x}) \approx \sum_{a=1}^{N_{t}} \phi^{a}(\mathbf{x}) \hat{u}^{a}, \quad \phi^{a}(\mathbf{x})=\sum_{\alpha, \gamma=1}^{m} p_{\alpha}(\mathbf{x}) \mathrm{A}_{\alpha \gamma}^{-1}(\mathbf{x}) \mathrm{B}_{\gamma}^{a}(\mathbf{x})
$$

where

$$
\mathrm{A}_{\alpha \beta}(\mathbf{x})=\sum_{a=1}^{N_{t}} w^{a}(\mathbf{x}) p_{\alpha}\left(\mathbf{x}^{a}\right) p_{\beta}\left(\mathbf{x}^{a}\right), \quad \mathrm{B}_{\alpha}^{a}(\mathbf{x})=w^{a}(\mathbf{x}) p_{\alpha}\left(\mathbf{x}^{a}\right) .
$$

The weight function for each node $\mathbf{x}^{a}$ is chosen as a function with a compact support given by the radius $r^{a}$. In this paper, we shall consider Gaussian weight functions:

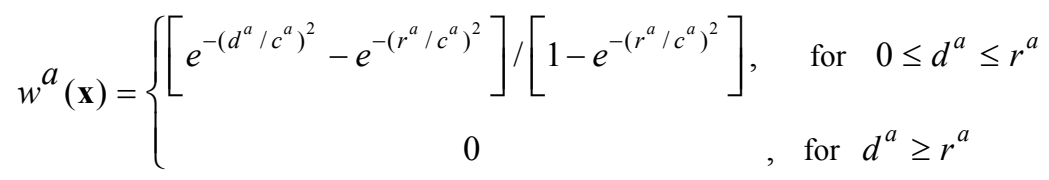

$N_{t}$ is the total number of nodes, but the actual number of nodes contributing to the approximation (7) is less than $N_{t}$, since $\phi^{a}(\mathbf{x})=0$, if $w^{a}(\mathbf{x})=0$. Nevertheless, all the $N_{t}$ nodes are involved into the evaluation algorithm for the shape functions. Recall that the shape functions do not satisfy the Kronecker delta property $\phi^{a}\left(\mathbf{x}^{b}\right) \neq \delta_{a b}$, in general, and the expansion coefficients $\hat{u}^{a}$ are fictitious nodal values. These nodal unknowns are discrete degrees of freedom in the discretized formulation.

\subsection{MLS-CAN concept}

Besides the standard MLS-approximation, we shall consider also the Central Approximation Node (CAN) concept. Let $\mathbf{x}^{q}$ be the CAN for the approximation at a point $\mathbf{x}$. Then, the amount of nodes involved into the approximation at $\mathbf{x}$ is reduced a-priori from $N_{t}$ to $N^{q}$, where $N^{q}$ is the number of nodes supporting the approximation at the CAN $\mathbf{x}^{q}$, i.e. the amount of nodes in the set $\mathcal{M}^{q}=\left\{\forall \mathbf{x}^{a} ; w^{a}\left(\mathbf{x}^{q}\right)>0\right\}_{a=1}^{N_{t}}$. Then, instead of the approximation given by Eq. (7), we shall use

$$
u(\mathbf{x})=\sum_{\alpha=1}^{N^{q}} \hat{u}^{n(q, \alpha)} \phi^{n(q, \alpha)}(\mathbf{x})
$$


where $n(q, \alpha)$ is the global number of the $\alpha$-th local node from $\mathcal{M}^{q}$. In this paper, we shall specify the CAN $\mathbf{x}^{q}$ as the nearest node to the approximation point $\mathbf{x}$.

\subsection{Approximation of potential field derivatives}

The gradients of the potential field can be approximated as gradients of the approximated potential by

$$
u_{, j}(\mathbf{x}) \approx \sum_{a=1}^{N_{t}} \phi_{, j}^{a}(\mathbf{x}) \hat{u}^{a}, \quad u_{, j}(\mathbf{x}) \approx \sum_{\alpha=1}^{N^{q}} \hat{u}^{n(q, \alpha)} \phi_{, j}^{n(q, \alpha)}(\mathbf{x}) .
$$

Note that calculation of gradients of the shape functions is rather complex procedure according to the formula

$$
\begin{aligned}
\phi_{, j}^{a}(\mathbf{x}) & =\sum_{\alpha, \gamma=1}^{m} p_{\alpha, j}(\mathbf{x}) \mathrm{A}_{\alpha \gamma}^{-1}(\mathbf{x}) \mathrm{B}_{\gamma}^{a}(\mathbf{x})+ \\
& +\sum_{\alpha, \gamma=1}^{m} p_{\alpha}(\mathbf{x})\left[\mathrm{A}_{\alpha \gamma}^{-1}(\mathbf{x}) \mathrm{B}_{\gamma, j}^{a}(\mathbf{x})-\sum_{\beta, \mu=1}^{m} \mathrm{~A}_{\alpha \beta}^{-1}(\mathbf{x}) \mathrm{A}_{\beta \mu, j}(\mathbf{x}) \mathrm{A}_{\mu \gamma}^{-1}(\mathbf{x}) \mathrm{B}_{\gamma}^{a}(\mathbf{x})\right] .
\end{aligned}
$$

The higher order derivatives can be obtained in a similar way

$$
u_{, j \ldots k}(\mathbf{x})=\sum_{\alpha=1}^{N^{q}} \hat{u}^{n(q, \alpha)} \phi_{, j \ldots k}^{n(q, \alpha)}(\mathbf{x})
$$

with increasing complexity of the evaluation. For instance, the second order derivatives are required in meshless implementation of the strong formulation given by the governing PDE (1). According to experience we know that the accuracy of higher order derivatives fails.

Beside the standard differentiation (referred as sdif), we can express the higher order derivatives of the potential field in terms of the first order derivatives of the shape functions $F_{k}^{c a}=\phi_{, k}^{(c, a)}\left(\mathbf{x}^{c}\right)$ and the nodal values $\hat{u}^{n(c, \alpha)}$ using the recurrent formula

$$
u_{, j j \ldots k}^{(\alpha)}\left(\mathbf{x}^{c}\right)=\sum_{a=1}^{N^{c}} u_{, j j \ldots}^{(\alpha-1)}\left(\mathbf{x}^{n(c, a)}\right) \phi_{, k}^{(c, a)}\left(\mathbf{x}^{c}\right)
$$

where the superscript $(\alpha)$ shows the order of the derivative. Thus,

$$
u_{, i j}\left(\mathbf{x}^{c}\right)=\sum_{a=1}^{N^{c}} F_{j}^{c a} \sum_{\substack{b=1 \\ v=n(c, a)}}^{N^{v}} F_{i}^{v b} \hat{u}^{n(v, b)}
$$




$$
u_{, i j k}\left(\mathbf{x}^{c}\right)=\sum_{a=1}^{N^{c}} F_{k}^{c a} \sum_{\substack{b=1 \\ v=n(c, a)}}^{N^{v}} F_{j}^{v b} \sum_{\substack{d=1 \\ w=n(v, b)}}^{N^{w}} F_{i}^{w d} \hat{u}^{n(w, d)} \text {, etc. }
$$

Note that Eqs. (14) and (15) can be rewritten as

$$
\begin{gathered}
u_{, i j}\left(\mathbf{x}^{c}\right)=\sum_{a=1}^{M^{c}} F_{i j}^{c a} \hat{u}^{m(c, a)}, \\
u_{, i j k}\left(\mathbf{x}^{c}\right)=\sum_{a=1}^{N^{c}} F_{k}^{c a} \sum_{\substack{b=1 \\
v=n(c, a)}}^{M^{v}} F_{i j}^{v b} \hat{u}^{m(v, b)}=\sum_{a=1}^{K^{c}} F_{i j k}^{c a} \hat{u}^{k(c, a)},
\end{gathered}
$$

where the global numbers $m(c, a)$ as well as $M^{c}$ and $F_{i j}^{c a}$ can be obtained from comparison of Eqs. (16) and (14). Similarly from (17) and (15), one can find $k(c, a), K^{c}$ and $F_{i j k}^{c a}$. These approaches will be referred as modified differentiation (mdif).

\section{Discretization of governing equations and boundary conditions}

\subsection{Strong formulation}

Collocating the prescribed boundary conditions at boundary nodes and the governing equations

$$
\lambda_{i k}\left(\mathbf{x}^{c}\right) u, k i\left(\mathbf{x}^{c}\right)+\lambda_{i k, i}\left(\mathbf{x}^{c}\right){ }_{, k}\left(\mathbf{x}^{c}\right)=-Q\left(\mathbf{x}^{c}\right),
$$

at interior nodal points $\mathbf{x}^{c}$ with substituting the approximations for the potential field and their gradients discussed in Sect. 3, we obtain the system of algebraic equations for the nodal unknowns. The numerical results achieved by this approach will be referred as CPDE (Collocation of PDE).

\subsection{Weak formulation}

Strictly speaking we present a mixed formulation, since the boundary conditions are considered in strong form. Surrounding each interior node $\mathbf{x}^{c}$ with a subdomain $\Omega^{c}$ and substituting an approximation for gradients of potential field at each integration point, one can complete the system of algebraic equations for computation of nodal unknowns $\hat{u}^{a}$ by equations

$$
\sum_{a=1}^{N_{t}} \hat{u}^{a} \int_{\partial \Omega^{c}} n_{i}(\mathbf{x}) \lambda_{i k}(\mathbf{x}) \phi_{, k}^{a}(\mathbf{x}) d \Gamma(\mathbf{x})=-\int_{\Omega^{c}} Q(\mathbf{x}) d \Omega(\mathbf{x}) .
$$


In Eq. (19), we have used the standard MLS approximation, but the MLSCAN approximation is applicable too. In general, the integrations are to be performed numerically with evaluating the gradients of the shape functions at each integration point.

In order to reduce the amount of points at which the shape function gradients are to be evaluated, we try to accomplish the integration in a closed form. For this purpose, we shall assume the circular sub-domains centred at nodes $\mathbf{x}^{c}$. Furthermore, the radius of the circle $r_{o}$ is taken sufficiently small, in order to justify the Taylor series expansion of the material coefficients as well as gradients of the shape functions within the sub-domain. If the material coefficients are prescribed by analytical functions, there is no basic problem to calculate their derivatives at nodal points. Expecting failure of accuracy of higher order derivatives of the shape functions, the size of the radius of sub-domains should guarantee fast convergence of the Taylor series expansion. For the sake of simplicity, we shall consider isotropic medium $\lambda_{i j}=\delta_{i j} \lambda$. Assuming the Taylor series expansions up to $6^{\text {th }}$ and $4^{\text {th }}$ orders for $\lambda(\mathbf{x})$ and $\phi_{, i}(\mathbf{x})$, respectively, and neglecting the terms $O\left(r_{o}^{8}\right)$, one obtains

$$
\begin{aligned}
& \frac{1}{\pi r_{o}^{2}} \int_{\partial \Omega^{c}} n_{i}(\mathbf{x}) \lambda(\mathbf{x}) u_{, i}(\mathbf{x}) d \Gamma=\left(\lambda_{, i}^{c}+\frac{r_{o}^{2}}{8} \lambda_{, i m m}^{c}+\frac{r_{o}^{4}}{24} \frac{1}{8} \lambda_{, i m m s s}^{c}\right) u_{, i}\left(\mathbf{x}^{c}\right)+ \\
& +\left[\lambda^{c} \delta_{i p}+\frac{r_{o}^{2}}{8}\left(2 \lambda_{, i p}^{c}+\lambda_{, j j}^{c} \delta_{i p}\right)+\frac{r_{o}^{4}}{24}\left(\frac{1}{2} \lambda_{, i p j j}^{c}+\frac{\delta_{i p}}{8} \lambda_{, s s j j}^{c}\right)+\right. \\
& \left.+\frac{r_{o}^{6}}{256}\left(\frac{1}{6} \lambda_{, i p j j s s}^{c}+\frac{\delta_{i p}}{36} \lambda_{, s s j j l l}^{c}\right)\right] u_{, i p}\left(\mathbf{x}^{c}\right)+\left[\frac{r_{o}^{2}}{8} 3 \lambda_{, i}^{c} \delta_{p s}+\frac{r_{o}^{4}}{24}\left(\frac{3}{4} \lambda_{, i j j}^{c} \delta_{p s}+\frac{1}{2} \lambda_{, i p s}^{c}\right)+\right. \\
& \left.+\frac{r_{o}^{6}}{256}\left(\frac{1}{4} \lambda_{, i j j l l}^{c} \delta_{p s}+\frac{1}{3} \lambda_{, i s p j j}^{c}\right)\right] u_{, i p s}\left(\mathbf{x}^{c}\right)+\left[\frac{r_{o}^{2}}{8} \lambda^{c} \delta_{i p} \delta_{k f}+\frac{r_{o}^{4}}{24}\left(\lambda_{, i p}^{c} \delta_{k f}+\frac{1}{4} \lambda_{, j j}^{c} \delta_{i p} \delta_{k f}\right)+\right. \\
& \left.+\frac{r_{o}^{6}}{6 \times 24 \times 192}\left(72 \lambda_{, i p j j}^{c} \delta_{k f}+24 \lambda_{, i p k f}^{c}+9 \lambda_{, j j l l}^{c} \delta_{i p} \delta_{k f}\right)\right] u_{, i p k f}\left(\mathbf{x}^{c}\right)= \\
& =-\frac{1}{\pi r_{o}^{2}} \Omega^{c} Q(\mathbf{x}) d \Omega(\mathbf{x})
\end{aligned}
$$

In the derivation of this equation, we have utilized the following integrals

$$
\frac{1}{r_{o}} \int_{\partial \Omega^{c}} n_{i} n_{j} d \Gamma=\int_{0}^{2 \pi} n_{i} n_{j} d \omega=\pi \delta_{i j},
$$




$$
\begin{aligned}
& \int_{0}^{2 \pi} n_{i} n_{j} n_{m} n_{p} d \omega=\frac{\pi}{4}\left(\delta_{i j} \delta_{m p}+\delta_{i m} \delta_{j p}+\delta_{i p} \delta_{j m}\right) \equiv Y_{i j m p}, \\
& \begin{aligned}
\int_{0}^{2 \pi} n_{i} n_{j} n_{m} n_{p} n_{t} n_{s} d \omega= & \frac{\pi}{24}\left(\delta_{i j} Y_{m p t s}+\delta_{i m} Y_{j p t s}+\delta_{i p} Y_{j m t s}+\delta_{i t} Y_{j m p s}+\delta_{i s} Y_{j m p t}\right) \equiv \\
\equiv & Y_{i j m p t s} \\
\int_{0}^{2 \pi} n_{i} n_{j} n_{m} n_{p} n_{t} n_{s} n_{r} n_{l} d \omega & =\frac{\pi}{192}\left(\delta_{i j} Y_{m p t s r l}+\delta_{i m} Y_{j p t s r l}+\delta_{i p} Y_{j m t s r l}+\delta_{i t} Y_{j m p s r l}+\right. \\
& \left.+\delta_{i s} Y_{j m p t r l}+\delta_{i r} Y_{j m p t s l}+\delta_{i l} Y_{j m p t s r}\right)
\end{aligned}
\end{aligned}
$$

and the integrals of the product of odd number of normal vectors are vanishing. Note that in the discretized form, the weak formulation given by Eq. (20) converges to the strong formulation (18) in the limit $r_{o} \rightarrow 0$. Moreover, the strong formulation corresponds to the lowest expansion terms in the weak formulation when the material coefficients and the shape functions gradients are expanded into Taylor series. Hence, one can expect better accuracy by the weak formulation than by the CPDE approach especially for problems in considerably graded materials.

\section{Numerical experiments}

Let us consider the square domain $L \times L$ with prescribed temperatures on the bottom and top of domain as $u_{o}$ and $u_{L}$, respectively, and thermally insulated lateral sides. The material medium is assumed to be isotropic with exponentially graded heat conduction coefficient as $\lambda(\mathbf{x})=\lambda_{o} \exp \left(n \delta x_{2} / L\right)$ with $n=2$ and $\delta=1$. The benchmark solution is given by the exact solution of this $1-\mathrm{d}$ problem

$$
u\left(x_{2}\right)=u_{o}+\frac{u_{L}-u_{o}}{e^{-n \delta}-1}\left(e^{-n \delta x_{2} / L}-1\right) .
$$

Before investigating the accuracy and efficiency of various meshless implementations of LIE and/or PDE, we discuss shortly the accuracy of approximations for derivatives of the potential field.

It can be seen from Fig. 1 that the approximation of the first order derivative is acceptable within the whole domain, while the accuracy for the second derivatives fails near the boundary. This can be explained by non-symmetric distribution of nodes w.r.t. the evaluation point in boundary layers. The sdif approach fails completely even in the case of the third derivative, while the mdif approach works well at points far from the boundary.

Fig. 2(a) shows the accuracy of numerical results achieved by analytical integrations and using the sdif approach for approximation of shape function derivatives with the highest order either two $(s f d o=2)$ or three $(s f d o=3)$. The $h$ parameter is the distance of two nearest nodes in uniform meshes, and the 

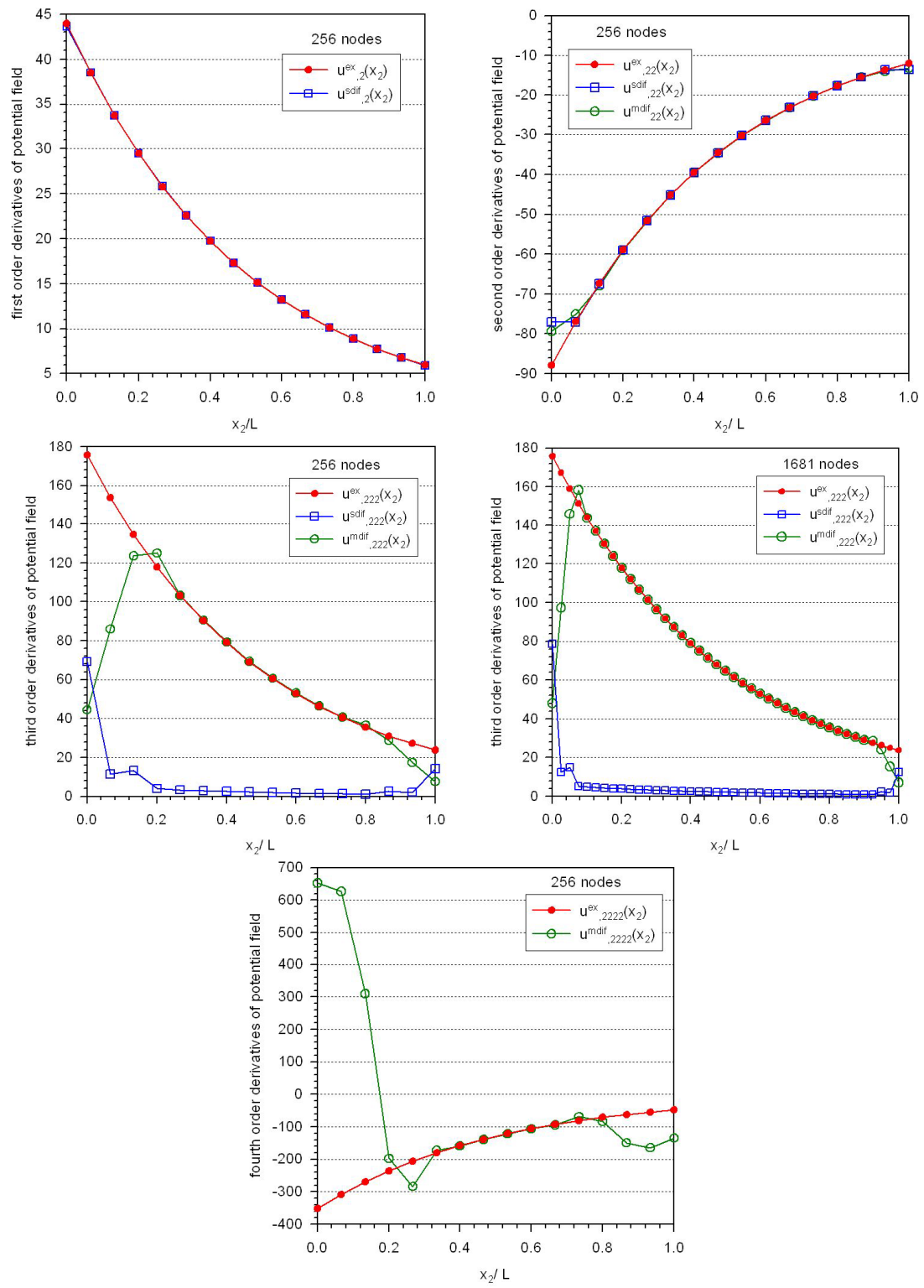

Figure 1: Comparison of approximated derivatives of potential with exact values.

radius of circular sub-domains was taken either independent on $h(r s d=0.01)$ or variable $(r s d=0.01 * h)$. One can see the negative influence of the third order derivative on accuracy. In the case of variable $r s d$, this influence is depressed because of minimization of the third order contribution with decreasing $r s d$ as $h$ is decreasing. 
In the case of LIE combined with the analytical integration, we have used various programming techniques for calculation of derivatives of shape functions within mdif approach. Although there was no influence on the accuracy, there was on the efficiency visible through the CPU times. When $r s d=0.01 * h$, the accuracy is independent on the choice of the highest order of the shape function derivatives owing to vanishing contribution of higher order terms with decreasing the radius of sub-domain. On the other hand, the influence of the sfdo is visible when $r s d=0.01$. The best accuracy as well as the convergence rate is achieved for $s f d o=3$. The less accurate results correspond to $s f d o=4$ because of worse approximations of the $4^{\text {th }}$ order derivatives of shape functions.

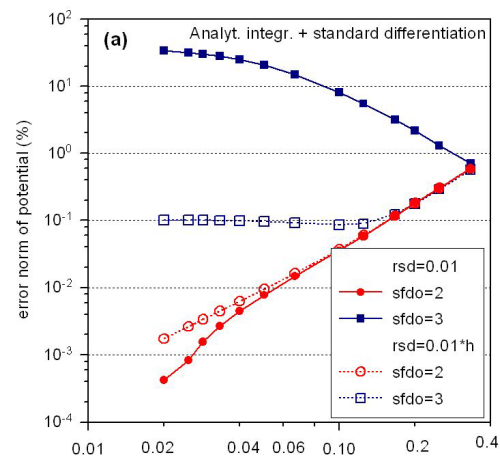

$\mathrm{h} / \mathrm{L}$

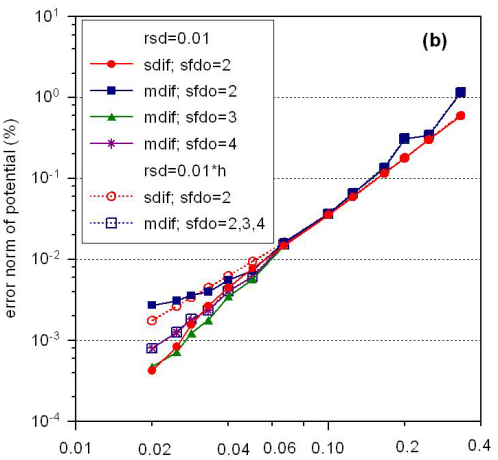

$\mathrm{h} / \mathrm{L}$

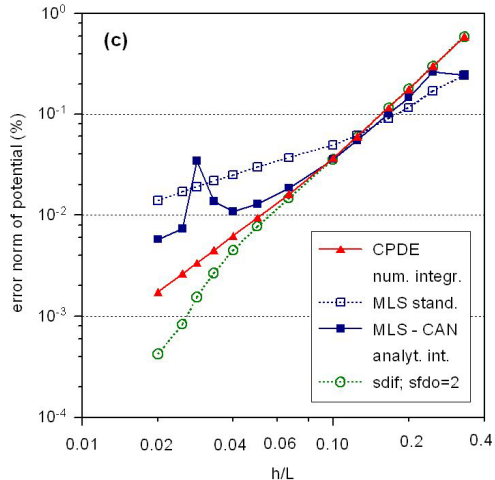

Figure 2: Convergence study for various techniques: (a) analyt. integration with sdif; (b) analyt. integration with either sdif or mdif;

CPDE, LIE+numer. integration, LIE+analyt. integr.

Finally, from Fig. 2(c), one can see that the LIE approach combined with numerical integration yield the worst accuracy as well as the convergence rate in comparison with the CPDE and the LIE+analytical integration approaches. As regards the LIE+analytical integration approaches, the best accuracy is achieved by the $s$ dif $+(s f d o=2)$ technique and comparable results are obtained by the mdif $+(s f d o=3)$ technique. 
The efficiency of various meshless computational techniques will be assessed by studying the CPU-times needed for creation of the system matrix $\left(t_{m}\right)$ and for complete solution $\left(t_{f}\right)$, where $t_{f}=t_{m}+t_{s}$ and $t_{s}$ is time needed for solution of the system of algebraic equations. Fig. 3(a) is a confirmation of the expectation that the LIE approach with numerical integration is less effective than CPDE in creation of the system matrix. It can be seen that for nodal point distributions with low density $t_{m}$ is a substantial part of $t_{f}$, while for high densities $t_{m}$ is a negligible part of $t_{f}$ for CPDE in contrast to the LIE+num. integr. approach.
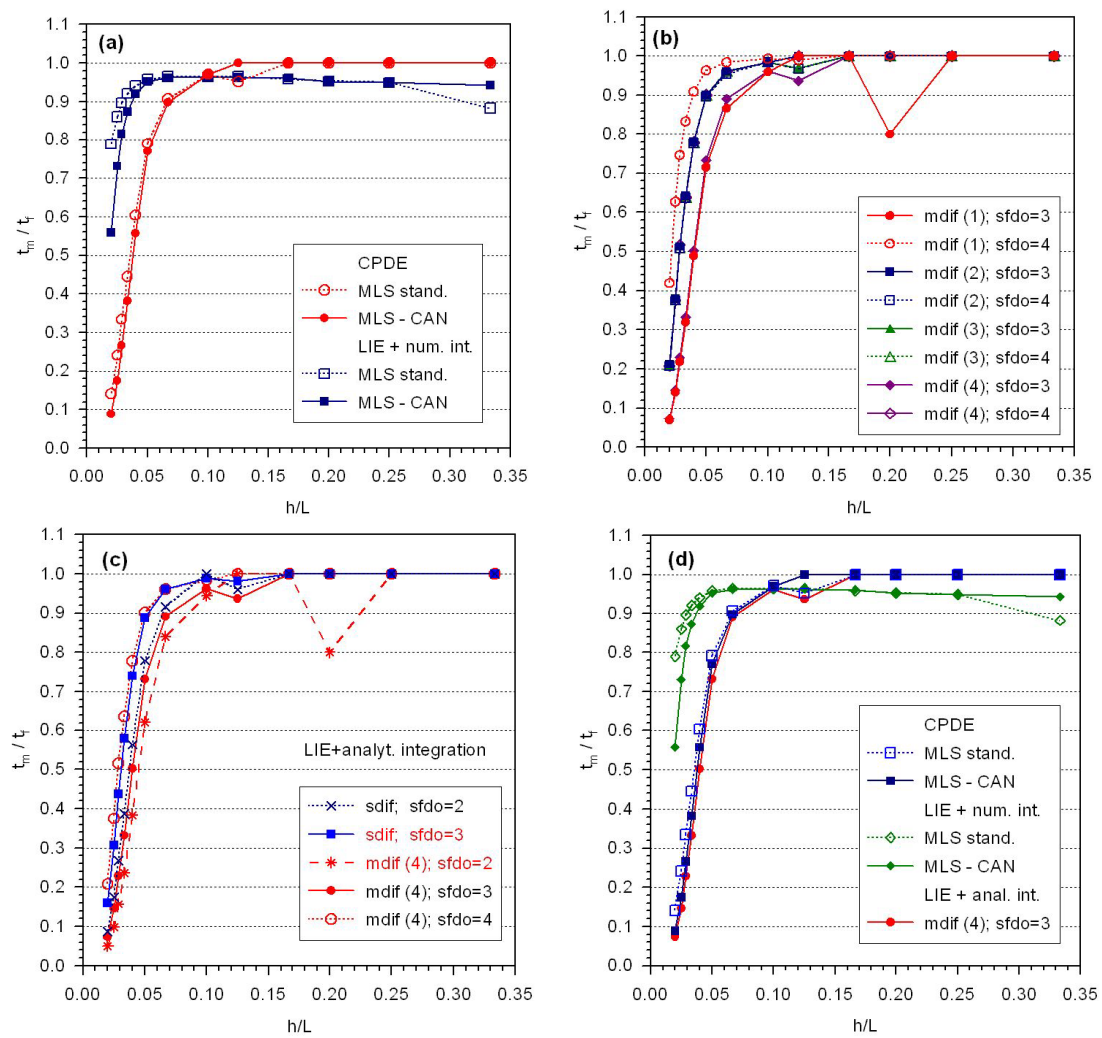

Figure 3: Variations of the time ratio $t_{m} / t_{f}$ with $h$ parameter for various meshless computational techniques.

Fig. 3(b) shows the dependence of the time ratio $t_{m} / t_{f}$ on $h$ parameter for LIE+analyt. integr. combined with four programming variants for modified calculation of shape function derivatives. Recall that all the variants mdif $(\alpha)$ with the same sfdo yield the same accuracy. The cases with $s f d o=2$ are not presented because of lower accuracy. It can be seen that the 
most effective variants are $\operatorname{mdif}(1)+(s f d o=3)$ and $\operatorname{mdif}(4)+(s f d o=3)$. In Fig. $3(c)$, we compare the efficiency of various approaches based on LIE+analytical integration. We presented also the results for $s d i f+(s f d o=3)$ and mdif $(4)+(s f d o=2)$ despite bad accuracy by these approaches. The approaches $s d i f+(s f d o=2)$ and $m$ dif $(4)+(s f d o=3)$ exhibit almost the same efficiency as well as the accuracy. Fig. 3(d) shows a comparison of efficiencies achieved by various meshless computational techniques.

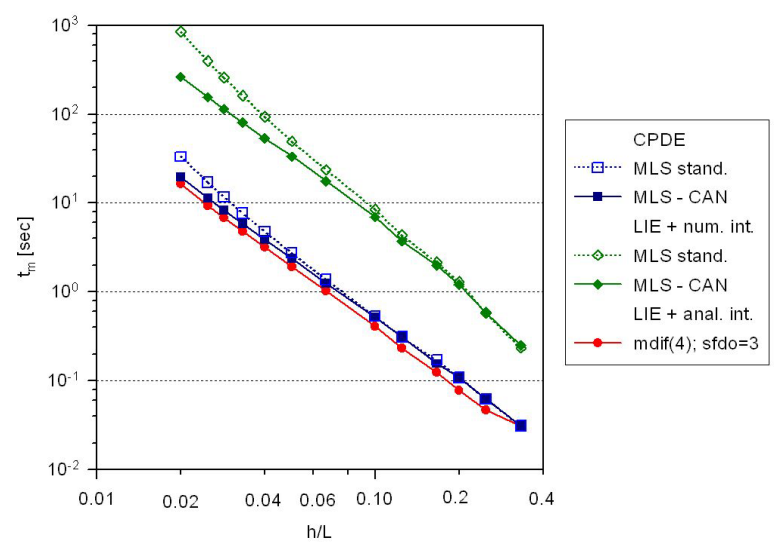

Figure 4: Dependence of $t_{m}$ on the $h$ parameter.

Finally, Fig. 4 shows the dependence of time needed for creation of the system matrix by various meshless computational techniques.

\section{Conclusions}

There was developed a meshless technique based on the LIE and analytical integration with effective computation of derivatives of shape functions. The efficiency in creation of the system matrix of discretized equations is comparable with the strong formulation based on the collocation of PDE at nodal points but the accuracy as well as the convergence rate is better in the proposed technique.

\section{References}

[1] Lancaster, P., Salkauskas, K., Surfaces generated by moving least square methods. Math. Comput., 37, pp. 141-158, 1981.

[2] Sladek, V., Sladek, J., Zhang, Ch., Computation of stresses in nonhomogeneous elastic solids by local integral equation method: a comparative study. Computational Mechanics, 41, pp. 827-845, 2008.

[3] Wrobel, L.C., The Boundary Element Method, Vol. 1: Applications in Thermo-Fluids and Acoustics, John Wiley \& Sons, Ltd., New York, 2002. 